\author{
Research Article
}

Olga Andreeva* and Valentina Malashchuk

\title{
The near-infrared observations at the Tower Solar Telescope TST-2 of the Crimean Astrophysical Observatory
}

https://doi.org/10.1515/astro-2021-0030

Received Nov 10, 2021; accepted Dec 29, 2021

\begin{abstract}
Observations in the near-infrared wavelength range have been regularly carried out at the Crimean Astrophysical Observatory (CrAO) on the Tower Solar Telescope (TST-2). It began in the late 1990s by putting into operation the Universal Spectrophotometer (USPh) at the TST-2 and it continues up to the present time. In 2020 the observation process was modernized - the mirror control electronics and software were replaced. Work is currently underway to create a catalog of solar disk images obtained before the modernization of TST-2. Here we present fragments of the catalog - several examples of a full solar disk in He i $1083 \mathrm{~nm}$ from the USPh/TST-2 instrument and a brief description of the future catalog.
\end{abstract}

Keywords: Sun, near-infrared spectroscopy, He I $1083 \mathrm{~nm}$ line

\section{Introduction}

Near-infrared spectroscopy is a modern instrumental method for quantitative and qualitative analysis of different objects, based on a combination of spectroscopy and statistical methods for investigating multifactorial dependencies. The infrared solar spectrum contains a wealth of physical data about our Sun, and is explored using modern detectors and technology with new ground-based solar telescopes. There are several advantages which can be realized by observing the Sun at infrared wavelengths compared to observations at shorter wavelengths (Penn 2014). These advantages concern the behavior of the Earth's atmosphere and the telescope, as well as the physics of atoms and solar radiative transfer. While these factors play an important role in studies of the solar disk, they can be absolutely critical for studies of the faint solar corona.

Corresponding Author: 0. Andreeva: Crimean Astrophysical Observatory, Russian Academy of Sciences, Nauchny, 298409, Russia; Email: olga.a7@gmail.com

V. Malashchuk: Crimean Astrophysical Observatory, Russian Academy of Sciences, Nauchny, 298409, Russia

\section{Infrared observations at the} TST-2/CrAO telescope

In the 1980s, works on the preparation of technical capabilities and software for observing in the near-infrared were started at the Crimean Astrophysical Observatory under the supervision of N.N. Stepanian. These observations in CrAO are carried out at the Tower Solar Telescope TST-2 (Figure 1) with the diffraction spectrograph and Universal Spectrophotometer (Stepanian et al. 2000).

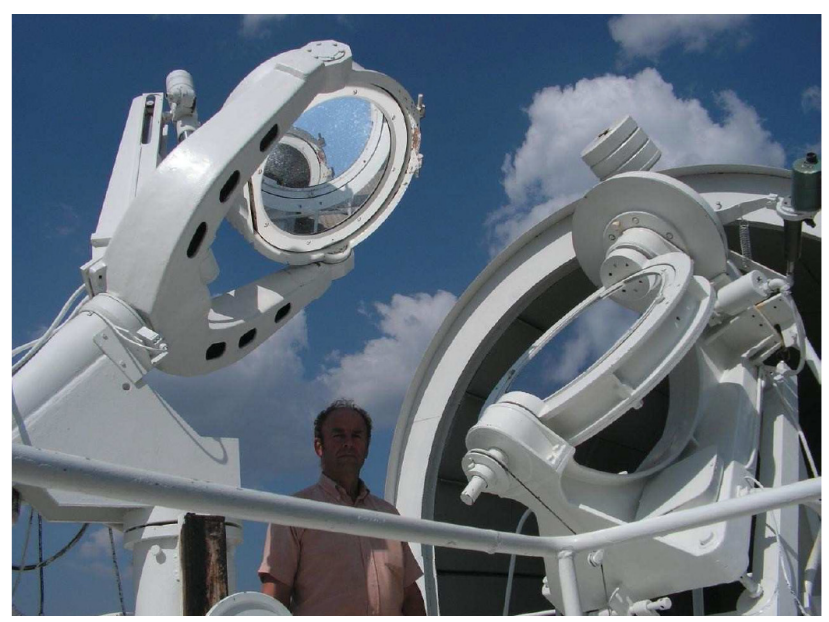

Figure 1. Coelostat of the Tower Solar Telescope TST-2 This work is licensed under the Creative Commons

๖ Open Access. ๑ 2021 0. Andreeva and V. Malashchuk, published by De Gruyter. Attribution 4.0 License 
By means of USPh it is possible to take images of the Sun in different spectral lines and record spectra of solar formations. The USPh scanner allows for shifting within two coordinates of the solar image at the spectrograph slit. The photomultiplier tube FEU-83 (Russ. abbr.) or CCD camera are used as a receiver at the spectrograph exit. The rather high sensibility of these receivers allows one to use USPh for studying the Sun in the near-infrared region, particularly, in the He I $1083 \mathrm{~nm}$ line (He I). The He I line is the strongest triplet neutral helium line, which makes it possible to study the physical properties of the upper chromosphere and the transition layer between the chromosphere and the corona. It is in this line, which is formed in the upper chromosphere at an altitude of about $2000-3000 \mathrm{~km}$ and is excited by ultraviolet radiation, that it is possible to observe coronal holes (CHs) from Earth. This line is the absorption line; in CHs it becomes weaker. The CHs in the He I line are brighter than the surrounding corona. The image (Figure 2) clearly shows coronal holes - the brightest formations, active regions - the darkest, and filaments - filamentous structures.

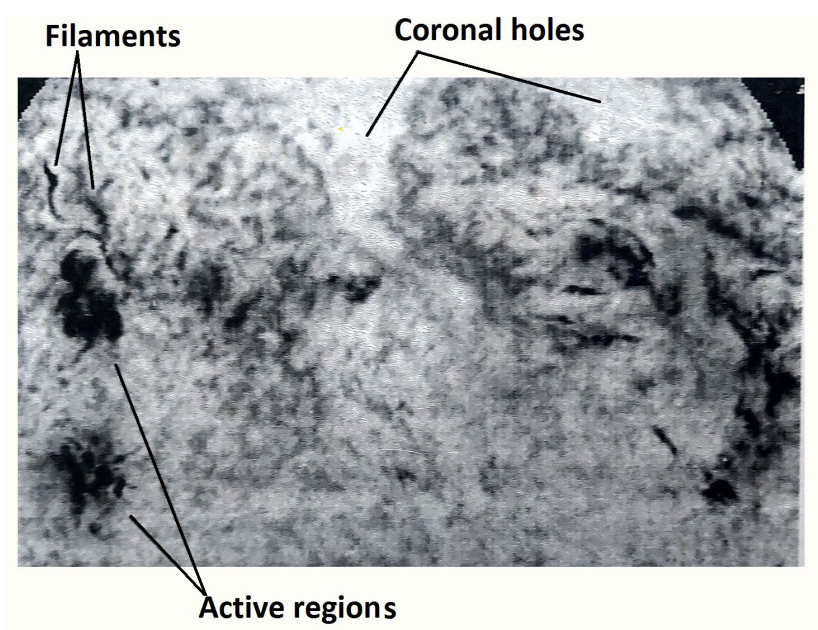

Figure 2. Solar structures on the image of the Sun obtained in the He I line

Service programs enable us, within several minutes to take into account limb darkening and analyze the image to identify CHs.

\section{Catalog excerpts}

The observations in the He I line have been carried out regularly since 1999 and are presented on the CrAO website https://sun.crao.ru/observations/izobrazheniyasolntsav-linii-hei-1083nm (one image per day). No observations were made from mid-2018 to the end of 2019. In 2020, the observation process and image processing were modernized. The mirror control electronics and software were changed. The observation methodology remained the same. A catalog of solar disk images obtained before the TST-2 telescope modernization, in 1999-2018 is currently being formed. It will also include images not shown on the website. All images are subject to unified processing.

Here are excerpts from the catalog (Figures 3, 4 and 5) and a brief description.The catalog contain files in FITS and GIF format. The FITS files represent the information recorded during image scanning: date and time of observation, spectral resolution, range of values observed, and angle B0.

The GIF file is represented by a map with a pair of images of the full-disk in He I $1083 \mathrm{~nm}$ line. The left image is unprocessed from the USPh/TST-2 instrument, the right image is processed with account of limb darkening. The maps also have image registration data and normalized to $2.4 \times 2.4$ arc seconds per pixel. A grid is superimposed on both images, on which the meridians and parallels are plotted at 10-degree intervals.

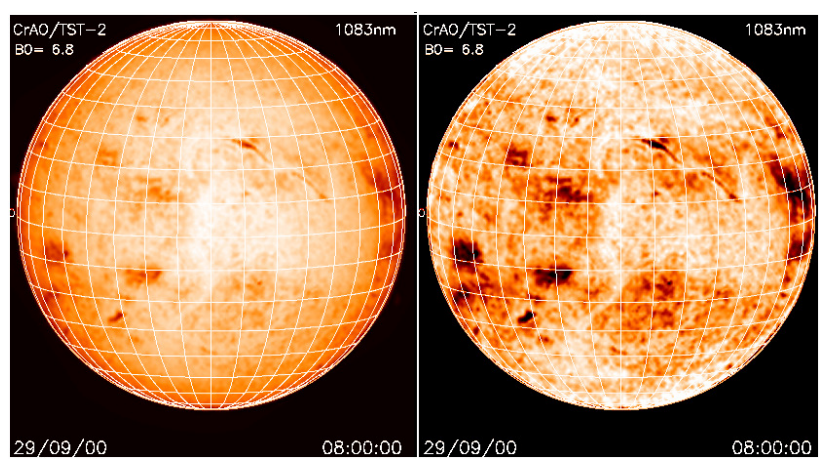

Figure 3. Full-disk images of the Sun obtained on September 29th, 2000 in the He I $1083 \mathrm{~nm}$ line (USPh/TST-2)

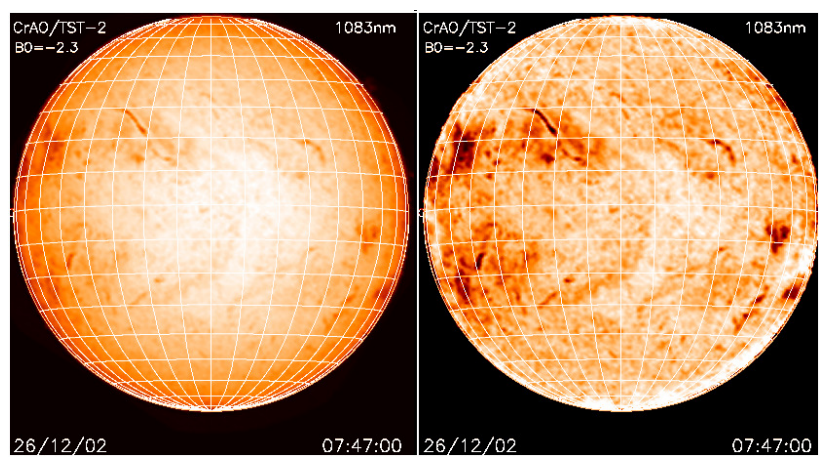

Figure 4. Full-disk images of the Sun obtained on December 26th, 2002 in the He I $1083 \mathrm{~nm}$ line (USPh/TST-2) 


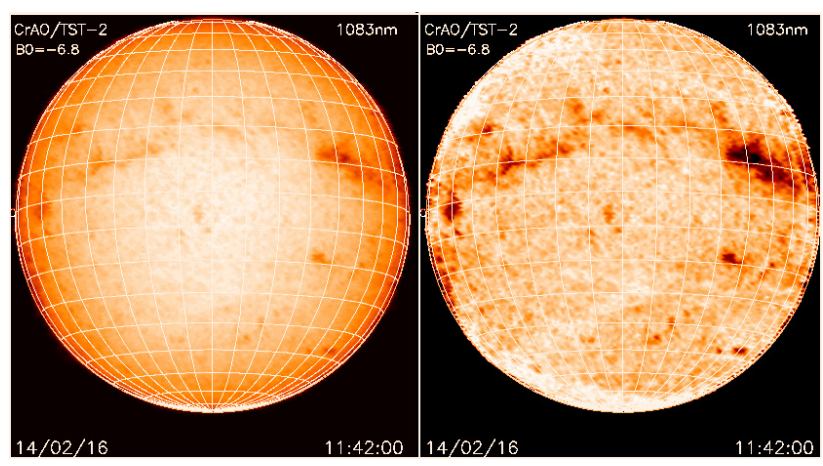

Figure 5. Full-disk images of the Sun obtained on February 14th, 2016 in the He I $1083 \mathrm{~nm}$ line (USPh/TST-2)

Figures 3, 4, and 5 show examples of a full solar disk maps obtained at different observational times (2000, 2002 and 2016, respectively) and reduced to the same view. Solar north is up on all maps. The right image of Figure 3 shows polar coronal holes as regions of less absorption, as well as an extended coronal hole crossing from the northern hemisphere to the southern hemisphere. Dark absorption accompanies active regions, and the quiet Sun shows less absorption, but reveals the internetwork pattern. A dark filament is visible on the disk in the northern hemisphere.

The catalog can be useful in carry out scientific research in the field of studying the nature and evolution of CHs, which contributes to the solution of such important problems of solar physics as the structure and physical conditions in the solar corona, rotation and evolution of large-scale magnetic fields, the formation of streams of the solar wind.
Funding information: The authors state no funding involved.

Author contributions: All authors have accepted responsibility for the entire content of this manuscript and approved its submission.

Conflict of interest: The authors state no conflict of interest.

\section{References}

Penn M. 2014. Infrared Solar Physics. Living Rev Solar Phys. 11(2):166. https://doi.org/10.12942//rsp-2014-2

Stepanian N, Dolgopolova E, Elizarov A, Malanushenko E, Parchevskii K, Sunitsa G. 2000. Solar universal spectrophotometer. Bull of the Crimean Astrophys Obs. 96:171-179. 